\title{
EFEITOS DA FAVA DE SUCUPIRA BRANCA (Pterodon pubescens) NA CICATRIZAÇÃO DE FERIDAS: REVISÃO INTEGRATIVA
}

\section{EFFECTS OF THE WHITE SUCUPIRA FAVA (Pterodon pubescens) ON WOULD HEALING: INTEGRATIVE REVIEW}

\author{
Fernanda de Souza ${ }^{1} *$ Priscilla Roberta Silva Rocha ${ }^{2}$
}

\begin{abstract}
RESUMO
Objetivo: buscar evidências na literatura sobre os benefícios da sucupira branca na cicatrização de feridas. Métodos: revisão integrativa de literatura, a busca foi realizada nas bases PubMed, Lilacs, Scielo, bem como na literatura cinzenta (Google Scholar). A estratégia de busca ocorreu conforme acrômio PICO, mediada pelos operadores booleanos AND e OR. Foram incluídos estudos desenvolvidos em animais, in vitro ou em humanos, sem limitação temporal. Os estudos foram classificados conforme nível de evidência. Resultados: 121 estudos foram recuperados, 09 foram lidos na integra e 06 foram selecionados para a revisão. De acordo com os dados obtidos, a atividade cicatrizante, anti-inflamatória, antirreumática, antiedematogênica e antinociceptiva do óleo da Pterodon pubescens é explicada pelos ativos geranilgerananiol, fração hexânica (ácido 6,7dihydroxyvouacapan-17-oic) e $\alpha$-humuleno. Conclusão: a espécie Pterodon pubescens parece ser benéfica para o uso no tratamento de feridas, entretanto há necessidade de pesquisas adicionais visando sua aplicação tópica, principalmente com foco na cicatrização, toxicidade, bem como mais testes em humanos.
\end{abstract}

Palavras Chaves: Pterodon pubescens; Fitoterapia; Cicatrização de Feridas; Terapêutica; Ferimentos e Lesões.

\begin{abstract}
Objective: to itenditfy the evidence in the literature about the benefits of Pterodon pubescens oil in wound healing. Methods: an integrative literature review, the search was performed in PubMed, Lilacs, Scielo databases, as well as in the gray literature (Google Scholar). The search strategy was done according to the PICO acromion, mediated by the Boolean operators AND and OR. Studies carried out in animals, in vitro or in humans, without temporal limitation, were included. Studies were classified according to the level of evidence. Results: 121 studies were retrieved, 09 were read in full and 06 were selected for review. According to the data obtained, the healing, antiinflammatory, antirheumatic, antiedematogenic and antinociceptive activity of Pterodon pubescens oil is explained by the actives geranilgerananiol, hexane fraction (6,7-dihydroxyvouacapan-17-oic acid) and $\alpha$-humulene. Conclusion: the species Pterodon pubescens seems to be beneficial for use in the treatment of wounds, however there is a need for further research aiming at its topical application, mainly focusing on healing, toxicity, as well as further testing in humans.
\end{abstract}

Keywords: Pterodon pubescens; Phytotherapy; Wound Healing; Therapeutics; Injuries and Wounds.

\footnotetext{
${ }^{1}$ Discente do curso de Graduação em Enfermagem da Facudade de Ceilândia, Universidade de Brasília, Campus Universitário s/n, Metropolitan Center, 72220-275, Brasília, DF, Brazil. Email: fernanda.de.ssouza@ gmail.com. ORCID: https://orcid.org/0000-00015202-0984

${ }^{2}$ Enfermeira, Doutora em Ciências da Saúde pela Universidade de Brasília, DF, Brasil. Professora adjunta do curso de enfermagem Universidade de Brasília - Faculdade de Ceilândia, Brasília, Brasil. E-mail: priscillarocha@unb.br. ORCID: https://orcid.org/00000002-2058-8548
} 


\section{INTRODUÇÃO}

As plantas têm um imenso potencial para o tratamento de feridas, são utilizadas há milênios por povos indígenas em muitos países. Os produtos à base de plantas são considerados econômicos, acessíveis e $\operatorname{seguros}^{(1)}$. Desde a Declaração de Alma-Ata, em 1978, a Organização Mundial da Saúde (OMS) tem expressado a sua posição a respeito da necessidade de valorizar a utilização de plantas medicinais no âmbito sanitário e na atenção básica à saúde ${ }^{(2)}$.

A OMS reconhece a fitoterapia como uma alternativa de tratamento viável com baixo custo e incentiva o levantamento, identificação, estímulo e orientação do uso das plantas medicinais que possuem eficácia e segurança terapêutica comprovada ${ }^{(3)}$. A Política Nacional de Práticas Integrativas e Complementares e a Política Nacional de Plantas Medicinais e Fitoterápicos (PPNPMF) estabelece diretrizes para garantir o acesso seguro e o uso racional das plantas medicinais e dos fitoterápicos no Brasil( ${ }^{(4)}$. Nos últimos anos os fitoterápicos ganharam espaço importante na reparação de tecidos, assim justificando o maior desdobramento científico em investigar essas alternativas ${ }^{(5,6)}$.

\section{As lesões tegumentares podem} apresentar etiologias distintas e características singulares. Após o dano o organismo inicia o processo de cicatrização e reparo tecidual, através de um processo complexo, composto por estágios interdependentes e simultâneos, envolvendo fenômenos químicos, físicos e biológicos, em três etapas básicas (inflamatória, proliferativa e remodelamento) $)^{(7,8)}$. Condições locais e sistêmicas influenciam o processo de reparo tecidual( ${ }^{(9)}$. Assim, a avaliação da lesão é primordial para a o planejamento terapêutico ${ }^{(10)}$.

O Brasil (um terço da flora mundial) apresenta uma rica biodiversidade, detêm uma vasta reserva de produtos naturais com potencial terapêutico. Dessa forma tem-se intensificado pesquisas sobre o uso desses fitoterápicos no processo de cicatrização de lesões cutâneas ${ }^{(11,12)}$. A fitoterapia ganha notoriedade ao explorar e valorizar recursos naturais do próprio bioma brasileiro, além do menor custo final e potencial terapêutico eficaz, aceitação cultural $\left.{ }^{(13},{ }^{14}\right)$.

Estudos em animais e in vitro sugerem que o óleo extraído da semente da Pterodon pubescens, conhecida como sucupira, sucupira-branca, faveiro, fava de sucupira, fava-de-santo-inácio e sucupira-lisa, pode auxiliar na cicatrização de feridas devido a ação anti-inflamatória, antiedematogênica e angiogênica ${ }^{(15-18)}$. Essa espécie encontra-se na região do Cerrado brasileiro, já sendo utilizada para tratamento de algumas doenças inflamatórias, reumáticas, dores de garganta e problemas respiratórios ${ }^{(19,20)}$. A atividade antiinflamatória é proveniente do extrato 
hexânico, constituído na sua formulação e atua através da inibição da liberação de prostaglandinas e outros mediadores envolvidos no sistema de cininas e compostos terpênicos ${ }^{(21)}$. Há também relatos da atividade analgésica e anti-inflamatória ${ }^{(22)}$, atividade $\operatorname{antinociceptiva}^{(23},{ }^{24)}$, atividade antimicrobiana, larvicida e de inibição da enzima acetilcolinesterase ${ }^{(25)}$.

Mediante tantas utilidades, desperta-se o interesse acerca da investigação sobre os efeitos da Pterodon pubescens no processo cicatricial. Este estudo teve como objetivo buscar evidências na literatura sobre os benefícios da sucupira branca na cicatrização de feridas.

\section{MÉTODOS}

Trata-se de uma revisão integrativa de literatura sobre uso da sucupira branca (Pterodon pubescens) no processo de cicatrização de lesões cutâneas. Para a elaboração da pergunta norteadora utilizou-se o acrônimo: Paciente, Intervenção, Comparação e Outcomes (desfecho), seguindo as etapas de uma revisão integrativa ${ }^{(26)}$.

A busca foi realizada em 14 de maio 2019, nas bases de dados U.S. National Library of Medicine National Institute of Health (Pubmed), Literatura LatinoAmericana e do Caribe em Ciências da Saúde (Lilacs) e Scientific Electronic Library Online (Scielo), utilizando os MESH terms
"Pterodon pubescens" AND "Phytotherapy" AND "Fatty Acids, Essential" AND "wound healing" OR "granulation tissue" OR "granulation tissues" OR "regeneration". A busca foi mediada pelos operadores booleanos AND para termos distintos e OR para termos similares. A busca na literatura cinzenta foi feita através do Google Scholar, utilizando os termos "Pterodon pubescens", "angiogenic", "wound healing" e "cicatrização".

Foram considerados os seguintes critérios de inclusão: os estudos desenvolvidos em animais, in vitro ou em humanos, sem limitação temporal de publicação, disponíveis em português, inglês e espanhol, que contemplem a intervenção estudada (uso da fava de sucupira branca) e o desfecho de interesse (a cicatrização de feridas). Foram excluídos os estudos não disponíveis integralmente, estudos secundários e aqueles que não atenderam a pergunta de pesquisa.

Os estudos encontrados foram exportados para o gerenciador de referências endnote web®, feita a exclusão eletrônica e manual das referências duplicadas. $\mathrm{Na}$ primeira fase (triagem), os estudos foram submetidos à leitura de título e resumo. Aqueles que atendiam os critérios de inclusão propostos, prosseguiram para a fase II. Estes foram submetidos a leitura integral para extração de dados; através de instrumento desenvolvido pelos próprios autores, foi 
coletado dos artigos incluídos nesta revisão as seguintes informações: identificação do artigo, ano de publicação, local de desenvolvimento do estudo, objetivo edesenho do estudo, nível de evidência, caracterização da amostra e fase da cicatrização estudada, resultados principais. Ambas as fases foram feitas por dois revisores independentes (F.S.S e P.R.S. R).

Os estudos foram classificados em níveis de evidência (NE): nível I - evidências procedentes de revisão sistemática ou metanálise de ensaios clínicos randomizados controlados relevantes ou originados de diretrizes clínicas baseadas em revisões sistemáticas de ensaios clínicos randomizados controlados; nível II - evidências obtidas de pelo menos um ensaio clínico randomizado controlado bem delineado; nível III evidências obtidas de ensaios clínicos bem delineados sem randomização; nível IV evidências oriundas de estudos de coorte e de caso-controle bem delineados; nível V - evidências originárias de revisão sistemática de estudos descritivos e qualitativos; nível VI - evidências procedentes de um único estudo descritivo ou qualitativo; nível VII evidências procedentes de opinião de autoridades e/ou relatório de comitês de especialistas $^{(27)}$.

\section{RESULTADOS}

Foram identificados 135 artigos a partir da busca nas bases citadas, não houve estudos duplicados. Os estudos foram submetidos a triagem através da leitura de título e resumo. Nove estudos foram selecionados para a leitura exploratória (leitura integral) e destes 06 foram selecionados para amostra final da revisão. Todos os estudos incluídos foram desenvolvidos no Brasil, sendo 66,7\% $(\mathrm{n}=4)$ na região sudoeste e $33,3 \%(n=2)$ na região centro oeste, conforme figura 1. Os estudos foram publicados entre os anos de 2002 a 2018, 66,7\% (n=4) foram publicados na língua portuguesa e $33,3 \%(n=2)$ na língua inglesa.

Figura I - Fluxograma segundo critérios de seleção dos estudos ${ }^{a}$. Brasília - DF, Brasil, 2020. 


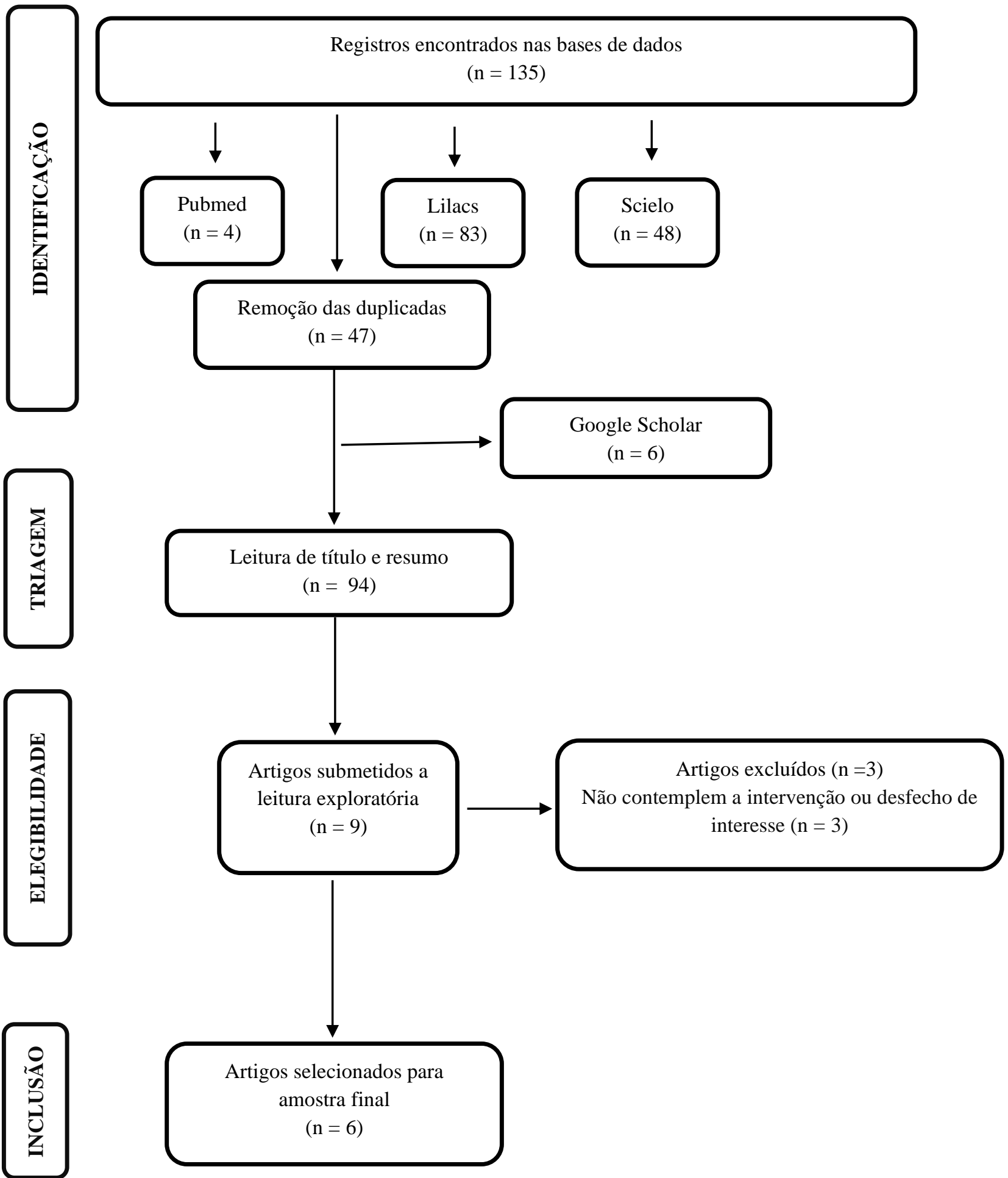

Fonte: Fluxograma adaptado Prisma

A maioria dos estudos apresentavam um componente experimental (in vitro ou in vivo $)^{(15,18,28,29)}$ e alguns estudos prosseguiram com ensaio clínico em animais (coelhos ou camundongos $)^{(15-17,28,29)}$. Nos experimentos in https://doi.org/10.31011/reaid-2021-v.95-n.36-art.1159 Rev Enferm Atual In Derme v. 95, n. 36, 2021 e-021153 vivo, foram induzidos intencionalmente processos inflamatórios para avaliar o comportamento da Pterodon pubescens e examinar possíveis mecanismos antiinflmatórios. Dentre as substâncias 5 
estimuladoras da inflamação utilizadas estão a carragenina $^{(15,28,29)}$, bradicinina ${ }^{(28)}$, óleo de cróton $^{(15,28)}$, histamina ${ }^{(28)}$ e PGE2 ${ }^{(29)}$. Houve também aindução inflamatória mecânica por queimadura através deplaca quente/barra de ferro ${ }^{(16,28,29)}$.

Nesta revisão, os principais efeitos do uso tópico do óleo essencial da Pterodon pubescens relatados consistem no aumento da quantidade de fibroblastos, na presença de atividade anti-inflamatória, antiedematogênica e angiogênica. Os estudos incluídos nessa revisão estão descritos na tabela 1 .

Os efeitos cicatrizantes da Pterodon pubescens foram comparados a substâncias que já possuem reconhecimento terapêutico convencional. As substâncias usadas como controle foram: a sulfadiazina de prata a $1 \%(16), \quad$ o $\quad$ Biocure ${ }^{\circledR} \quad$ (estimulador

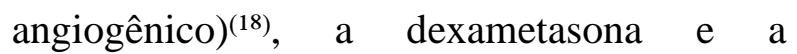
indometacina (anti-inflamatórios e antiedematogênicos) $)^{(28,29)}$, a ciproheptadina (anti-histamínico) $^{(28)}$ e o Dersani ${ }^{(17)}$.

A atividade anti-inflamatória pode ser explicada por ativos que consistem em sua fórmula, como a fração hexânica (composta principalmente pelo ácido 6,7dihydroxyvouacapan-17-oic, um diterpeno vuacapeno), o qual contribui para o estímulo de fibroblastos ${ }^{(16)}$, na redução do edema, além de possuir atividade anti-inflamatória $\left.{ }^{(15}, 16\right)$. Outros componentes da família dos terpenos como o geranilgerananiol e o farnesol também https://doi.org/10.31011/reaid-2021-v.95-n.36-art.1159 Rev Enferm Atual In Derme v. 95, n. 36, 2021 e-021153 desempenharam redução da resposta edematosa e inflamatória, ${ }^{(15)}$ com decaimento da atividade das prostaglandinas ${ }^{(21)}$ e da interleucina 8 (IL-8), também observada pelo $\alpha$-humuleno em teste in vitro ${ }^{(29)}$.

O $\alpha$-humuleno tem função angiogênica, pois em altas concentrações estimula o fator de crescimento endotelial vascular (VEGF), facilitando a migração e proliferação celular, contribuindo para o fechamento de lesões ${ }^{(29)}$. Os estudos incluídos na revisão avaliaram o uso da Pterodon pubescens associada a outras estratégias terapêuticas cicatrizantes ${ }^{(17)}$. $\mathrm{Na}$ avaliação concomitante da laserterapia com o óleo essencial de sucupira no tratamento de feridas de ratos diabéticos, foi demonstrada um comportamento sinérgico. Segundo os autores o extrato hexânico da Pterodon pubescens auxiliou no mecanismo anti-inflamatório e angiogênico, enquanto a laserterapia fomentou o processo de maturação do tecido com melhor distribuição e organização do colágeno $^{(17)}$.

Outra terapia associativa descrita nos estudos desta revisão, foi o uso do extrato bruto da Pterodon pubescens juntamente com óleo essencial da Cordia verbenacea DC, administrados por via oral ${ }^{(29)}$. Os autores constataram efeito anti-inflamatório e antinociceptivo em doses mais baixas, quando comparados ao uso dos extratos brutos isolados, não foi demonstrado efeitos colaterais $^{(29)}$. 
Embora a Pterodon pubescens tenha apresentado atividade antinociceptiva e antiinflamatória, vale ressaltar que dentre os estudos incluídos, um estudo relatou efeito tóxico relacionado ao uso oral da dose efetiva $(300 \mathrm{mg} / \mathrm{kg})$ após 14 dias $^{(28)}$. Foi observado discreto dano renal, gastrite e edema da submucosa devido a diminuição de prostaglandinas (principalmente PG2 e PGI2), hipoglicemia, diminuição de peso, degeneração celular e dano hepático pela interferência na eliminação da bilirrubina, além da atividade antiproliferativa em cultura de células tumorais, provavelmente relacionada a inibição de $\mathrm{COX}-2^{(28)}$.

Os estudos incluídos nesta revisão apontam que no óleo essencial, na sua fração bruta ou extratos isolados da sucupira, há principios ativos capazes de auxiliar no processo cicatricial na classe dos terpenos, como derivados vouacapânicos, geranilgeraniol, com destaque para a fração hexânica e $\alpha$-humuleno, gerando potencial terapêutico mais evidente nas fases inflamatória e proliferativa da cicatrização ${ }^{(15-}$ $18,28,29)$. 
Tabela 1 - Estudos incluídos na revisão, Brasília, 2020.

\begin{tabular}{|c|c|c|c|}
\hline $\begin{array}{l}\text { Identificação } \\
\text { e local do } \\
\text { estudo }\end{array}$ & $\begin{array}{l}\text { Objetivo do estudo } \\
\text { Tipo de estudo, nível de } \\
\text { evidência }\end{array}$ & $\begin{array}{l}\text { aracterização da amostra, descrição da intervenção, fase } \\
\text { cicatrização avaliada }\end{array}$ & Resultados principais \\
\hline $\begin{array}{l}\text { Juiz de Fora, } \\
\text { Minas Gerais. } \\
\text { Brasil }\end{array}$ & $\begin{array}{l}\text { Avaliar a cicatrização de } \\
\text { lesões cutâneas em coelhos, } \\
\text { tratados com cremes com } \mathrm{OE}^{2} \\
\text { e } \mathrm{FH}^{3} \text { derivadas de Pterodon } \\
\text { emarginatus } \\
\text { Ensaio Clínico (não } \\
\text { randominzação) } \\
\mathrm{NE} \text { : III }\end{array}$ & $\begin{array}{l}\text { Coelhos albinos da Nova Zelândia (machos, } 1,5-2,0 \mathrm{~kg}) \\
\text { divididos em } 2 \text { grupos:(1): OE a } 5 \text { e } 10 \% \text { e }(2) \text { : FH a } 10 \text { e } 20 \% \text {. } \\
\text { Dorso dividido em } 4 \text { quadrantes, cada qual recebeu um } \\
\text { tratamento diferente, } 2 \text { x por dia }(12 \mathrm{~h}-24 \mathrm{~h}) \text { durante } 10 \text { dias } \\
\text { consecutivos } \\
\text { OE e FH incorporados em creme base lanette, nas concentrações } \\
\text { (p/p): OE ( } 5 \text { e } 10 \%) \text { e FH (10 e } 20 \%) \text { Controle: creme de } \\
\text { sulfadiazina de prata } 1 \% \text { (controle positivo) e o creme base } \\
\text { lanette (controle negativo) }\end{array}$ & $\begin{array}{l}\text { Atividade cicatrizante: OE }(5 \text { e } 10 \%) \text { e } \mathrm{FH} 10 \% \text {; } \\
\text { redução da quantidade de células inflamatórias } \mathrm{FH}^{3} \\
(\mathrm{p}<0,05) \text {; aumento dos fibroblastos: OE } 5 \text { e } 10 \%, \mathrm{FH} \text { a } \\
10 \% \text { (p<0,01). Atividade angiogênica: OE } 10 \% \text {, FH } \\
20 \%(\mathrm{p}<0,01) \text { Hemostasia: sulfadiazina de prata (Q1), } \\
\text { OE 5\% (Q3) e 10\% (Q4), apresentaram diminuição nos } \\
\text { pontos de hemorragia. OE (5 e 10\%) e FH 10\% } \\
\text { apresentaram atividade cicatrizante, diminuição de } \\
\text { células inflamatórias, aumento de fibroblastos e vasos } \\
\text { sanguíneos. Nenhuma substância avaliada demostrou } \\
\text { alterações no colágeno da lesão. }\end{array}$ \\
\hline $\begin{array}{l}\text { De Araujo et } \\
\text { al }^{(18)} \\
\text { Brasil, } \\
\text { Goiânia/Goiás }\end{array}$ & 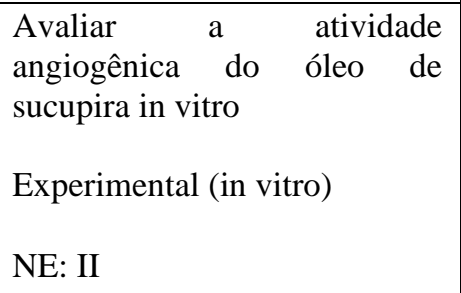 & $\begin{array}{l}40 \text { ovos (MCA) do embrião do pintiho (Gallus domesticus), } \\
\text { grupos: (1) Intervenção: Óleo de sucupira (Amazon Leve) a } \\
\text { 1g/ml; (2) Controle neutro: água destilada estéril; (3) Inibidor: } \\
\text { dexametasona 4mg/ml (inibidor); (4) Controle positivo: } \\
\text { Biomembrana de latéx (biocure) } \\
\text { Avaliação da angiogênese }\end{array}$ & $\begin{array}{l}\text { O óleo da fava de suc } \\
\text { induziu um aumento c } \\
\text { comparados aos gru } \\
\text { inibidor (dexametason } \\
\text { e controle positivo (B } \\
\text { indução da rede vascu }\end{array}$ \\
\hline pinas/ & 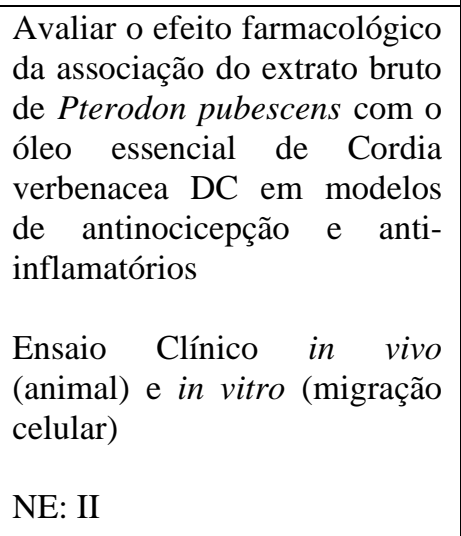 & $\begin{array}{l}\text { In vitro: Cultura de células: queratinócitos HaCaT não- } \\
\text { tumorigênicos humanos e In vivo: Camundongos Swiss fêmeas } \\
\text { submetidas a ativação da resposta inflamatória álgica e anti- } \\
\text { edematogênica. Grupos: } \\
\text { (1) Edema de pata por carragenina }(\mathrm{n}=6) \text { : Controle positivo } \\
\text { (dexametasona } 5 \mathrm{mg} / \mathrm{kg}) \text {; Controle negativo }(10 \mathrm{mg} / \mathrm{kg} \text { veículo+ } \\
\text { Tween®) } \\
\text { (2) Edema de pata por PGE2: Controle positivo (indometacina } \\
20 \mathrm{mg} / \mathrm{kg}) ; \text { Controle negativo }(10 \mathrm{mg} / \mathrm{kg} \text { veículo+ Tween®) } \\
\mathrm{Cv}+\mathrm{Pp} \text { nas VO: } \\
\text { A50 }(82,5 \mathrm{mg} / \mathrm{kg}+32,5 \mathrm{mg} / \mathrm{kg}) \text {, A100 }(165 \mathrm{mg} / \mathrm{kg}+65 \mathrm{mg} / \mathrm{kg}) \text {, } \\
\text { A200 }(330 \mathrm{mg} / \mathrm{kg}+130 \mathrm{mg} / \mathrm{kg}) \\
\text { Avaliada fase inflamatória e proliferativa }\end{array}$ & $\begin{array}{l}\text { A associação do Pp e Cv tem efeitos sinérgicos } \\
\text { antinociceptivos e anti-inflamatórios, redução do } \\
\text { edema (induzido pela carragenina a partir da } 4^{\circ} \text { hora } \\
\left.\text { até } 48^{\circ}\right)(p<0,001) \text {, redução do edema por PGE2 } 2^{10} \text { na } \\
\text { dose de A } 50(p<0,05) \text {, inibição da proliferação e a } \\
\text { migração celular in vitro (p <0.05). Em doses maiores } \\
(12,5-25 \mu \mathrm{M}) \text { o } \alpha \text {-Humuleno pode ter dupla atividade: } \\
\text { efeito anti-inflamatório (reduz a produção de IL-8) e } \\
\text { angiogênico (aumenta os níveis de VEGF), em mais } \\
\text { baixas }(6,25 \mu \mathrm{M}) \text {, atividade anti-angiogênica ( } \downarrow \\
\text { secreção de VEGF). }\end{array}$ \\
\hline Denr & $\begin{array}{l}\text { valiar a atividade anti- } \\
\text { aflamatória, antinociceptiva e }\end{array}$ & $\begin{array}{l}n \text { vitro: Células neoplásicas humanas (culturas) - pulmão, } \\
\text { nama e melanoma }\end{array}$ & croton) $1 \%$ ), \\
\hline
\end{tabular}




\begin{tabular}{|c|c|c|c|}
\hline $\begin{array}{l}\text { Brasil, } \\
\text { Piracicaba/ } \\
\text { São Paulo }\end{array}$ & $\begin{array}{l}\text { a toxicidade aguda do óleo das } \\
\text { sementes da Pterodon } \\
\text { pubescens e identificar a } \\
\text { fração ativa. } \\
\text { Ensaio clínico (randomizado), } \\
\text { e estudo experimental in vivo } \\
\text { e in vitro. } \\
\text { NE: I }\end{array}$ & $\begin{array}{l}\text { Camundongos albinos Mus músculos Swiss machos, adultos } \\
\text { (25-35g) e ratos Ratus novergicus Wistar machos adultos (100- } \\
220 \mathrm{~g} \text { ) adrenalectomizados. Grupos: } \\
\text { (1) Edema de pata por carragenina. Controle positivo: } \\
\text { indometacina } 10 \mathrm{mg} / \mathrm{kg} \text {; Controle negativo: } \mathrm{NaCl} 0,9 \% \\
\text { (2) Edema de pata por histamina. Controle positivo: } \\
\text { ciproheptadina } 50 \mathrm{mg} / \mathrm{kg} \text {; Controle negativo: } \mathrm{NaCl} 0,9 \% \\
\text { (3) Edema de orelha por óleo de cróton. } \mathrm{Controle} \text { positivo: } \\
\text { dexametasona } 1 \mathrm{mg} / \mathrm{kg} \text {; Controle negativo: } \mathrm{NaCl} 0,9 \% \\
\text { (4) Edema de pata por bradicinina. Controle positivo: ausente; } \\
\text { Controle negativo: } \mathrm{NaCl} 0,9 \%\end{array}$ & 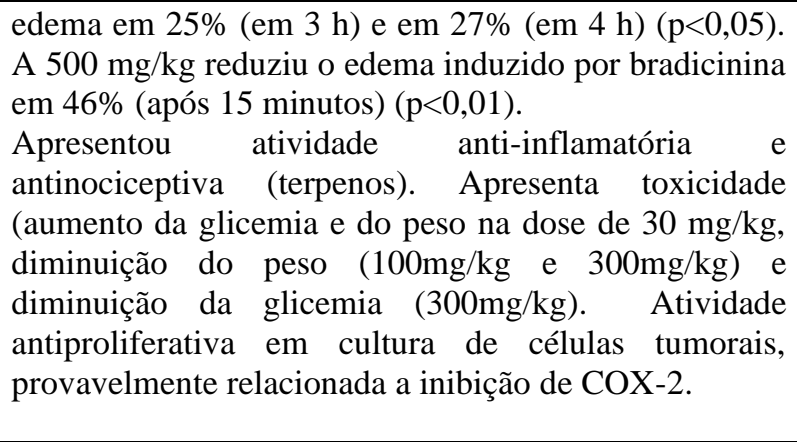 \\
\hline $\begin{array}{l}\text { Silva et al }{ }^{(15)} \\
\text { Brasil, Rio de } \\
\text { Janeiro }\end{array}$ & $\begin{array}{l}\begin{array}{l}\text { Avaliar se o extrato de } \\
\text { sementes de } P \text {. pubescens } \\
\text { possui } \\
\text { antiedematogênica aguda }\end{array} \\
\text { Ensaio Clínico randomizado } \\
\text { NE: I }\end{array}$ & $\begin{array}{l}\text { Camundongos SW machos (12 } \pm 15 \text { semanas de idade e } 30 \pm 40 \\
\text { g de peso corporal) } \\
\text { Grupo controle vs. Grupo intervenção com } \mathrm{OE}^{2} \text { em diferentes } \\
\text { frações [PF1 (29.89 g), PF2 }(23.89 \mathrm{~g}), \mathrm{PF} 3(0.23 \mathrm{~g}) \text { e PF4 } \\
\text { (resíduo, } 0.028 \mathrm{~g}) \text {. } \\
\text { O edema foi induzido pela injeção de carragenina e por óleo de } \\
\text { cróton } \\
\text { Avaliada fase inflamatória }\end{array}$ & $\begin{array}{l}\text { Efeito antiedematogênicas predominou na fração } \\
\text { hexânica, produzindo três subfrações (PF1.1, PF1.2 e } \\
\text { PF1.3). A PF1.1 e PF1.3 tem atividade tõpica } \\
\text { antiedematosa aguda potente }(\mathrm{p}<0.05) \text {. A PF1.2, não } \\
\text { foi ativa no ensaio de carragenina, mas exibiu potente } \\
\text { atividade antiedematogênica no edema na orelha } \\
\text { induzido por óleo de cróton (p<0.05). O extrato de } P p \\
\text { tem atividade antiedematogênica aguda e tópica. } \\
\text { Outros compostos além do vouacapan, como o } \\
\text { geranilgeraniol, contribuem para atividade anti- } \\
\text { inflamatória do OE. }\end{array}$ \\
\hline $\begin{array}{l}\text { Marra }^{(17)} \\
\text { Brasil. Distrito } \\
\text { Federal, } \\
\text { Brasília }\end{array}$ & $\begin{array}{l}\text { Avaliar o perfil cicatricial de } \\
\text { ratos diabéticos sob uso de } \\
\text { LDT-PPH isoladamente ou } \\
\text { precedido da aplicação de } \\
\text { laserterapia de baixa } \\
\text { intensidade } \\
\text { Ensaio clínico não } \\
\text { randomizado } \\
\text { NE: III }\end{array}$ & $\begin{array}{l}42 \text { ratos (Rattus novergicus - albino, linhagem Wistar, fêmeas } \\
\text { adultas com peso entre } 150 \text { e } 250 \text { gramas, induzidos a DM, } \\
\text { divididos em } 03 \text { grupos: } \\
\text { (1) Grupo Controle: animais sob o uso apenas do veículo } \\
\text { (Dersani }{ }^{\circledR)} \\
\text { (2) Grupo LDT-PPH } \\
\text { (3) Grupo LLLT + LDT-PPH }\end{array}$ & $\begin{array}{l}\text { LDT-PPH } 1 \% 2 \times \text { ao dia foi mais efetivo, promoveu } \\
\text { semelhança no ritmo de fechamento e melhor aspecto } \\
\text { do formato das feridas. Não houve diferença } \\
\text { macroscópica quanto ao fechamento da área entre os } \\
\text { grupos ao longo do período de observação. LDT-PPH } \\
\text { isolado ou em combinação com a laserterapia (LLLT + } \\
\text { LDT-PPH) aumentou a vascularização comparada ao } \\
\text { controle (p<0,05). A deposição colágena nos grupos } \\
\text { tratados com melhor aspecto do formato das feridas } \\
\text { mostrou diferença significativa na segunda semana de } \\
\text { tratamento em relação ao grupo controle. }\end{array}$ \\
\hline
\end{tabular}

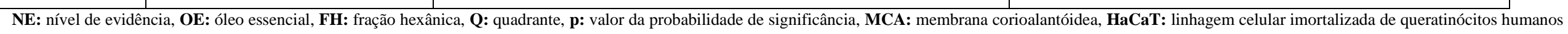

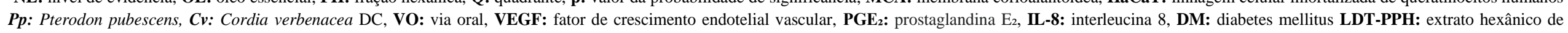

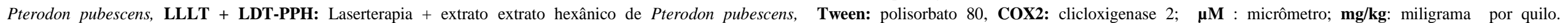




\section{DISCUSSÃO}

O uso de plantas medicinais se faz desde os primórdios da civilização como prática terapêutica empírica, perdurando até hoje principalmente pela população indígena e em regiões do interior ${ }^{(30)}$. Presume-se que as vantagens da fitoterapia sejam derivadas de interações sinérgicas que intensificam os seus efeitos farmacológicos, com possível redução de seus efeitos colaterais ${ }^{(31)}$. A Pterodon pubescens, popularmente conhecida como fava de sucupira, administrada por via oral ou intraperitoneal possui efeito antirreumático, antiedematogênico, antinociceptivo e antiinflamatório ${ }^{(15,21,32-41)}$, corroborando com os dados obtidos nesta revisão. Estudos que avaliam formulações para o uso tópico ainda são incipientes $\left.{ }^{(42}, 43\right)$, havendo necessidade de maiores investigações.

Os principais compostos isolados das espécies de Pterodon são diterpenos lineares e/ou tetracíclicos com esqueletos de vouacapano, que estão subjacentes em parte às atividades farmacológicas do óleo(44), Isoflavonas e sesquiterpenos também são encontrados no extrato oleaginoso ${ }^{(19)}$.

\section{Efeitos Analgésicos}

A ação analgésica decorrente da atividade anti-inflamatória pode ser explicada pela presença de ativos terpênicos como a fração hexânica (composta majoritariamente por $6 \alpha, 7 \beta$-dihydroxy-vouacapan-173-oic) $)^{(35)}$.

Quando administrada por via oral, mostrou inibição dos receptores vanilóides VR1, receptores periféricos de glutamato, capsaicina e na ativação de vias inibitórias serotoninérgicas ${ }^{(33},{ }^{34)}$, elucidando assim seus mecanismos de ação.

O geranilgerananiol consiste em um outro componente da $P$. pubescens, funcionando contra estados de dor possivelmente relacionados às vias glutamato, capsaicina, sistema serotoninérgico $\left(5-\mathrm{HT}_{3}\right)$ e imidazolina $\left(\mathrm{I}_{1}\right)$, tendo resposta positiva a circunstâncias que causam hiperarlgesia ${ }^{(33},{ }^{34)}$. Assim ambos os compostos, ativos terpênicos e o geranilgerananiol, possuem respostas antialodínica, ou seja, reduz a produção de estímulos dolorosos que em situações normais não geram dor ${ }^{(34)}$ até mesmo decorrente de dor pós-operatória ${ }^{(37)}$. Os mecanismos descritos, tornam a semente de $P$. pubescens bastante promissora no tratamento de distúrbios da dor, com o efeito atribuível aos derivados vouacapanos ${ }^{(37,45)}$, fortalecendo assim os achados de estudos prévios ${ }^{(29) .}$

\section{Efeitos anti-inflamatório}

\section{antiedematogênico}

Os mecanismos antiedematoso antiinflamatório corroboram os achados de 
estudos incluídos nesta revisão, que descrevem o papel antiedematoso tópico e o efeito anti-inflamatório em ratos adrenalectomiazados $^{(15,28)}$. $\mathrm{O}$ experimento sugere que a ação do óleo deva ser independente da liberação de corticoides endógenos. Outro componente da fração hexânica, os derivados de vouacapan, contribuíram positivamente para a prevenção de edema em camundongos, com efeito imunomodulador, podendo beneficiar pessoas com condições inflamatórias crônicas como a artrite reumatoide ${ }^{(15,32,38)}$.

A ação anti-inflamatória da fava de sucupira está relacionada à presença de compostos terpenos já citados ${ }^{(15,34,45)}$ que por outro mecanismo de ação, possui respostas anti-inflamatórias e antiálgicas observadas nos ensaios in vivo como inibidor da COX$2^{(41)}$.

\section{Ação angiogênica e cicatrizante}

Nesta revisão foi reportado a ação antiinflamatória e a atividade cicatrizante da fração hexânica (rica no composto ácido 6 $\alpha, 7 \beta$-dihidroxivouacapano-17 $\beta$-óico). Há relatos da atividade cicatrizante do óleo essencial nas doses $5-10 \%$ e $10 \%$, ação angiogênica nas doses $10 \%$ e $20 \%$, fibroplásica nas doses $5-10 \%$ e $10 \%$ e hemostática nas doses $5 \%$ e $10 \%{ }^{(16)}$. Os estudos incluídos demonstram que o $\alpha$ humuleno auxilia no aumento de fibroblastos, diminuição de IL-8 e em altas doses (12$25 \mu \mathrm{M})$ acresce os níveis do fator de crescimento endotelial vascular (VEGF), importante no processo de angiogênese ${ }^{(18,29)}$.

Perante todas estas propriedades, torna-se interessante a veiculação deste óleo por uso tópico na cicatrização de feridas. A Pterodon pubescens se torna uma estratégia potencial para a cicatrização de lesões cutâneas, especialmente na fase inflamatória pela ação da fração hexânica e geranilgerananiol e da fase proliferativa devido a atividade do $\alpha$ humuleno, possibilitando a utilização do fitoterápico em seu extrato bruto, óleo essencial ou uma fração dos seus princípios ativos.

\section{Perspectivas}

Uma fórmula padrão ainda não foi firmada para o emprego específico em feridas de humanos, mas há estudos que analisaram formulação para seu uso tópico em camundongos ${ }^{(42},{ }^{43)}$. A estabilidade envolvendo o extrato bruto de $P$. pubescens indicou que o gel de Carbopol contendo 2,5\% de extrato vegetal bruto livre apresentou além de melhores características organolépticas, valores de $\mathrm{pH}$ compatíveis com o da pele e estabilidade do produto de $180 \operatorname{dias}^{(42)}$. 
Vale ressaltar que o $\mathrm{pH}$ do leito da ferida geralmente difere com o da pele íntegra, a lesão apresenta um meio ácido como uma resposta fisiológica temporária(46), o qual beneficia o processo de cicatrização. A associação do ácido hialurônico com lipossomas promove o aumento da permeabilidade cutânea, facilitando a absorção da Pterodon pubescens à pele(47). Entretanto, são necessários mais estudos acerca da estabilidade e durabilidade dos compostos contendo Pterodon pubescens.

Os achados dessa revisão mostram a efetividade da Pterodon pubescens no tratamento das feridas ao contribuir no processo cicatricial por meio da redução de edema (devido ação anti-inflamatória), angiogênese e fibroplasia.

\section{Toxicidades}

Alguns estudos reportam toxicidades relacionados ao uso da Pterodon pubescens. A fração hexânica na dose $250 \mathrm{mg} / \mathrm{kg}$ (via oral) reduziu níveis de glicose, colesterol e triglicerídeos, fornecendo uma base racional para o uso do extrato de sucupira na medicina popular $^{(38)}$. Por outro lado, esse composto nas doses de 100 e $300 \mathrm{mg} / \mathrm{kg}$ (oral) foi associado a diminuição do peso corporal, vale ressaltar que na dose de $300 \mathrm{mg} / \mathrm{kg}$ apresentou resultado hipoglicemiante ${ }^{(28)}$. Este efeito foi reafirmado em novas abordagens para a gestão de Diabetes Mellitus, no qual foi proposto o encapsulamento do óleo essencial sucupira $^{(48)}$.

Embora os achados de um estudo clínico incluído na revisão ${ }^{(28)}$ tenha observado a ocorrência de esplenomegalia, gastrite, nefrite e degeneração hepática nos animais tratados com a dose $300 \mathrm{mg} / \mathrm{kg}$ por via intraperitoneal, o extrato bruto ou a fração hexânica do óleo da Pterodon pubescens não se mostrou tóxico em testes macroscópicos, hematológicos, bioquímicos, anatomopatológicos e de medidas do peso ${ }^{(13,38,49,50)}$. Essa discordância poderia ser explicada pela via de administração empregada, já que nos estudos apresentados a via oral foi escolhida e quando se optou pela via intraperitoneal, houve sinais de toxicidade, porém se faz necessários estudos para afirmar esta proposição. Assim faz-se necessário estudos adicionais que testem formulações que possam ser aplicadas em feridas, que avaliem a toxicidade, estabilidade, durabilidade e periodicidade de aplicação tópica do óleo da Pterodon pubescens, visto suas comprovadas ações terapêuticas.

As limitações desta pesquisa foram: a escassez de estudos sobre o uso tópico de 
Pterodon pubescens no processo cicatricial em humanos. Apenas um dos estudos incluídos aborda toxicidades ${ }^{(28)}$, há relatos de vias de administração distintas (oral e peritoneal), bem como diferentes concentrações utilizadas nos estudos. Os achados reforçam os efeitos benéficos da fava de sucupira, entretanto são necessários novos estudos especialmente para o tratamento de lesões cutâneas.

\section{CONSIDERAÇÕES FINAIS}

Os achados desta revisão evidenciam que a fava de sucupira branca apresenta atividades anti-inflamatória, cicatrizante, antirreumática, antiedematogênica e antinociceptiva comprovadas cientificamente. Estas propriedades se dão pelos ativos presentes, como a fração hexânica, rica em 6,7dihydroxyvouacapan-17-oic,

geranilgerananiol e o $\alpha$-humuleno. A fava de sucupira branca apresentam potencial terapêutico no processo cicatricial de feridas, se fazendo necessário maiores estudos por via tópica em animais e testes em humanos.

\section{REFERÊNCIAS}

1. Thakur R, Jain N, Pathak R, Sandhu SS. Practices in Wound Healing Studies of Plants. Evidence-Based Complementary and em 12 de agosto de 2020]; 2011:438056. Disponível https://doi.org/10.1155/2011/438056 Alternative Medicine [Internet]. 2011[acesso

2. Organización Mundial de la Salud. Estrategia de la OMS sobre medicina tradicional 20022005. Geneva: Organización Mundial de la Salud; 2002.

3. Ministério da Saúde (BR). Portaria n ${ }^{\circ}$ 971, de 03 de maio de 2006. Aprova a Política Nacional de Práticas Integrativas e Complementares (PNPIC) no Sistema Único de Saúde. Diário Oficial da União. 3 maio; 2006.

4. Maver T. Maver U, Stana KK, Smrke DM, Kreft S. A review of herbal medicines in wound healing. International journal of dermatology [Internet]. 2015 [acesso em 12 de agosto de 2020]; 54(7):740-757. Disponível em: https://doi.org/10.1111/ijd.12766

5. Barros NF. Política Nacional de Práticas Integrativas e Complementares no SUS: uma ação de inclusão. Cien Saude Colet [Internet]. 2006 [acesso em 12 de agosto de 2020]; 11(3):850. Disponível em: http://dx.doi.org/10.1590/S141381232006000300034

6. Ferreira KFC, Nardin JM. Avaliação in vitro da citotoxicidade do extrato de Bauhínia glabra em células linfocitárias normais. Caderno da Escola de Saúde [Internet]. 2015 [acesso em 12 de agosto de 2020]; 1(13):7992. Disponível em: https://portaldeperiodicos.unibrasil.com.br/ind ex.php/cadernossaude/article/view/2430

7. Lindley LE, Stojadinovic O, Pastar I, TomicCanic M. Biology and Biomarkers for Wound Healing. Plast Reconstr Surg [Internet]. 2016 [acesso em 12 de agosto de 2020]; 138(3Suppl):18S-28S. Disponível em: https://doi.org/10.1097/PRS.00000000000026 82

8. Childs DR, Murthy AS. Overview of Wound Healing and Management. Surg Clin North https://doi.org/10.31011/reaid-2021-v.95-n.36-art.1159 Rev Enferm Atual In Derme v. 95, n. 36, 2021 e-021153 
Am [Internet]. 2017 [acesso em 12 de agosto de 2020]; 97(1):189-207. Disponível em: http://dx.doi.org/10.1016/j.suc.2016.08.013

9. Han G, Ceilley R. Chronic Wound Healing: A Review of Current Management and Treatments. Adv Ther [Internet]. 2017 [acesso em 12 de agosto de 2020]; 34(3):599-610. Disponível em: https://doi.org/10.1007/s12325-017-0478-y

10. Sorg H, Tilkorn DJ, Hager S, Hauser J, Mirastschijski U. Skin Wound Healing: An Update on the Current Knowledge and Concepts. Eur Surg Res [Internet]. 2017 [acesso em 12 de agosto de 2020]; 58(12):81-94. Disponível em: https://doi.org/10.1159/000454919

11. Yunes RA, Pedrosa RC, Cechinel-Filho V. Fármacos e fitoterápicos: a necessidade do desenvolvimento da indústria de fitoterápicos e fitofármacos no Brasil. Química Nova [Internet]. 2001 [acesso em 12 de agosto de 2020]; 24(1):147-152. Disponível em: https://doi.org/10.1590/S010040422001000100025

12. França ISX, Souza JA, Baptista RS, Brito VRS. Medicina popular: benefícios e malefícios das plantas medicinais. Revista Brasileira de Enfermagem [Internet]. 2008 [acesso em 12 de agosto de 2020]; 61(2):201$8 . \quad$ Disponível em: https://doi.org/10.1590/S003471672008000200009

13. Sabino KC, Gayer CR, Vaz LC, Santos LR, Felzenszwalb I, Coelho MG. In vitro and in vivo toxicological study of the Pterodon pubescens seed oil. Toxicol Lett [Internet]. 1999 [acesso em 12 de Agosto de 2020]; 108(1):27-35. Disponível em: https://doi.org/10.1016/S03784274(99)00110-1
14. Pinto Coelho MG, Marques PR, Gayer CR, Vaz LC, Neto JF, Sabino KC. Subacute toxicity evaluation of a hydroalcoholic extract of Pterodon pubescens seeds in mice with collageninduced arthritis. J Ethnopharmacol [Internet]. 2001[acesso em 12 de agosto de 2020]; 77(2-3):159-64. Disponível em: https://doi.org/10.1016/S0378-

8741(01)00288-4

15. Silva MC, Gayer CR, Lopes CS, Calixto NO, Reis PA, Passaes CP, Paes MC, Dalmau SR, Sabino KC, Todeschini AR, Coelho MGP. Acute and topic anti-edematogenic fractions isolated from the seeds of Pterodon pubescens. J Pharm Pharmacol [Internet]. 2004 [acesso em 12 de agosto]; 56(1):135141. Disponível em: https://doi.org/10.1211/0022357022485

16. Dutra RC, Pittella F, Ferreira AS, Larcher P, Farias RE, Barbosa NR. Efeito cicatrizante das sementes de Pterodon emarginatus Vogel em modelos de úlceras dérmicas experimentais em coelhos. Latin American Journal of Pharmacy [Internet]. 2009 [acesso em 12 de agosto de 2020]; 28(3):375-382. Disponível em: http://www.latamjpharm.org/trabajos/28/3/LA JOP_28_3_1_8_0O4O1V30HG.pdf

17. Marra, Ana Aparecida. Wound healing profile of diabectic rats in the use of LDT-PPH and low level laser therapy, 2010. 10f. Dissertação (Mestrado em Ciências da Saúde) Universidade Católica de Brasília, Brasília, 2010.

https://bdtd.ucb.br:8443/jspui/handle/1234567 89/1124 [incluído na revisão]

18. De Araújo, LA, Assunção LA, Junior NJ. Da S, Lemes SR, Reis PR, De M. Angiogenic activity of sucupira (Pterodon emarginatus) oil. Scientia Medica [Internet]. 2015 [acesso em 12 de agosto de 2020]; 25(2):ID20351. Disponível 
http://dx.doi.org/10.15448/1980-

6108.2015.2.20351

19. Arriaga AMC, MAB De Castro, ER Silveira e R. Braz-Filho. Outros diterpenóides isolados de Pterodon polygalaeflorus. Jornal da Sociedade Brasileira de Química [Internet]. 2000 [acesso em 12 de agosto de 2020]; 11(2):187-190. Disponível em: https://doi.org/10.1590/S010350532000000200015

20. Lorenzi H, Matos FJA Matos. Plantas medicinais no Brasil: nativas e exóticas. Instituto Plantarum Ed. Nova Odessa, SP; 2002.

21. Carvalho JCT, Sertié JAA, Barbosa MVJ, Patrício KCM, Caputo LRG, Sarti SJ, Ferreira, L.P, Bastos JK. Anti-inflammatory activity of the crude extract form the fruits of Pterodon emarginatus Vog. Journal of ethnopharmacology [Internet]. 1999 [acesso em 12 de agosto de 2020]; 64(2):127-33, $1999 . \quad$ Disponível em: https://doi.org/10.1016/s03788741(98)00116-0

22. Belinelo VJ, Reis GT, Stefani GM, Ferreira Alves D-L, Piló-Veloso D. Synthesis of 6 $\alpha, 7 \beta$-Dihydroxyvouacapan-17 $\beta$-oic Acid Derivatives. Part IV: Mannich Base Derivatives and its Activities on the Electrically Stimulated. J Braz Chem Soc [Internet]. 2002 [acesso em 12 de Agosto de 2020]; 13(6):830-837. Disponível em: https://doi.org/10.1590/S0103-

50532002000600016

23. Leal LKAM, Ferreira AG, Bezerra GA, Matos FJA, Viana GSB. Antinociceptive, anti-inflammatory and bronchodilator activities of Brazilian medicinal plants containing coumarin: a comparative study. Journal of Ethnopharmacology [Internet]. 2000 [acesso de 12 de Agosto de 2020]; https://doi.org/10.31011/reaid-2021-v.95-n.36-art.1159 Rev Enferm Atual In Derme v. 95, n. 36, 2021 e-021153
70(2):151-159. Disponível

em: https://doi.org/10.1016/S03788741(99)00165-8

24. Evangelista GL, Coelho-de-Souza AN, Santos CF, Leal-Cardoso JH, Lopes EA, dos Santos MV, Lahlou S, Magalhães PJ. Essential oil of Pterodon polygalaeflorus inhibits electromechanical coupling on rat isolated trachea. J Ethnopharmacol [Internet]. 2007 [acesso em 12 de agosto de 2020]; 109(3):515-22. Disponível em: https://doi.org/10.1016/j.jep.2006.08.028

25. Pimenta ATA, Santiago GMP, Arriaga AMC, Menezes GHA, Bezerra SB. Estudo fitoquímico e avaliação da atividade larvicida de Pterodon polygalaeflorus Benth (Leguminosae) sobre Aedes aegypti. Revista Brasileira de Farmacognosia [Internet]. 2006 [acesso em 12 de agosto de 2020]; 16(4):501$505 . \quad$ Disponível em: https://doi.org/10.1590/S0102695X2006000400011

26. Palaskar JN. Framing the research question using PICO strategy. J Dent Allied Sci [Internet]. 2017 [acesso em 12 de agosto de 2020]; 6:55. Disponível em: https://doi.org/10.4103/jdas.jdas_46_17

27. Melnyk BM, Fineoutoverholt E. Making the case for evidence-based practice. In: Evidence-based practice in nursing \& healthcare: a guide to best practice [CDROOM]. Philadelphia: Lippincott Willians Wilkins; 2005. p. 324.

28. Denny, Carina. Atividade anti-inflamatória do óleo de sucupira: Pterodon pubenscens Benth Leguminosae-papilionoideae [dissertação]. São Paulo: Faculdade de Odontologia de Piracicaba da Universidade Estadual de Campinas, Piracicaba; 2002. Disponível em: http://repositorio.unicamp.br/jspui/handle/RE POSIP/289320 [incluído na revisão]

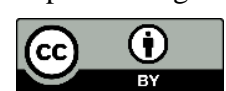


29. Basting, RT. Avaliação da associação do extrato bruto diclorometânico de frutos de Pterodon pubescens Benth. e do óleo essencial de Cordia verbenacea DC na atividade antinociceptiva e anti-inflamatória [tese]. São Paulo: Faculdade de Ciências Médicas da Universidade Estadual de Campinas; 2018. Disponível em: http:://repositório.unicamp.br/jspui/handle/RE POSIP/333660

30. Pazyar N, Yaghoobi R, Rafiee E, Mehrabian A, Feily A. Skin wound healing and phytomedicine: A review. Skin Pharmacol Physiol [Internet]. 2014 [acesso em 12 de agosto de 2020]; 27(6):303-310. Disponível em: https://doi.org/10.1159/000357477

31. Hildebert W, Ulrich-Merzenich G. Synergy research: Appproaching a new generation of phytopharmaceuticals. Phytomedicine [Internet]. 2009 [acesso em de 12 de agosto de 2020]; 16(2-3):97-110. Disponível em: https://doi.org/10.1016/j.phymed.2008.12.018

32. Coelho MG, Sabino KC, Dalmau SR. Immunomodulatory effects of sucupira (Pterodon pubescens) seed infusion on collagen-induced arthritis. Clin Exp Rheumatol [Internet]. 2004 [acesso em 12 de agosto de 2020]; 22(2):213-218. Disponível em:

https://pubmed.ncbi.nlm.nih.gov/15083889/

33. Spindola HM, Servat L, Denny C, Rodrigues RA, Eberlin MN, Cabral E, Sousa IM, Tamashiro JY, Carvalho JE, Foglio MA. Antinociceptive effect of geranylgeraniol and 6alpha,7beta-dihydroxyvouacapan-17betaoate methyl ester isolated from Pterodon pubescens Benth. BMC Pharmacol [Internet]. 2010 [acesso em 12 de agosto de 2020]; 10:1. Disponível em: https://doi.org/10.1186/14712210-10-1

34. Spindola HM, Servat L, Rodrigues RA, Sousa IM, Carvalho JE, Foglio MA. Geranylgeraniol and $6 \alpha, 7 \beta-$ dihydroxyvouacapan-17 $\beta$-oate methyl ester isolated from Pterodon pubescens Benth.: Further investigation on the antinociceptive mechanisms of action. Eur $\mathrm{J}$ Pharmacol [Internet]. 2011 [acesso em 12 de agosto de 2020]; 656(1-3):45-51. Disponível em: https://doi.org/10.1016/j.ejphar.2011.01.025

35. Servat, L. Atividade antinociceptiva e anticâncer in vitro de microencapsulados produzidos com extrato bruto e vouacapanos obtidos das sementes de Pterodon pubescens Benth [dissertação]. São Paulo: Faculdade de Odontologia de Piracicaba da Universidade Estadual de Campinas; 2010. Disponível em: http://www.repositorio.unicamp.br/bitstream/ REPOSIP/288230/1/Servat-

Medina_Leila_M.pdf

36. Servat L, Spindola HM, Rodrigues RAF, Sousa IMO, Ruiz ALTG, Carvalho JE, Foglio MA. Pterodon pubescens Benth.: stability study of microencapsulated extract and isolated compounds monitored by antinociceptive assays. J. Braz. Chem Soc [Internet]. 2012 [acesso em 12 de agosto de 2020]; 23(7):1244-1253. Disponível em: https://doi.org/10.1590/S010350532012000700008

37. Nucci C, Mazzardo-Martins L, Stramosk J, Brethanha LC, Pizzolatti MG, Santos AR, Martins DF. Oleaginous extract from the fruits Pterodon pubescens Benth induces antinociception in animal models of acute and chronic pain. J Ethnopharmacol [Internet]. 2012 [acesso em 12 de agosto de 2020]; 143(1):170-178. Disponível em: https://doi.org/10.1016/j.jep.2012.06.020

38. Hoscheid J, Bersani-Amado CA, da Rocha BA, Outuki PM, da Silva MA, Froehlich DL, Cardoso ML. Inhibitory Effect of the Hexane Fraction of the Ethanolic Extract of the Fruits of Pterodon pubescens Benth in Acute and Chronic Inflammation. Evid Based Complement Alternat Med [Internet]. 2013 
[acesso em 12 de agosto de 2020]; https://doi.org/10.1155/2013/272795

39. Santos ÉS, Garcia FP, Outuki PM, Hoschield J, Goes PRN, Cardozo-Filho L, Nakamura CV, Cardoso MLC. Optimization of extraction method and evaluation of antileishmanial activity of oil and nanoemulsions of Pterodon pubescens benth. fruit extracts. Exp Parasitol [Internet]. 2016 [acesso em 12 de agosto de 2020]; 170:252$260 . \quad$ Disponível em: https://doi.org/10.1016/j.exppara.2016.10.004

40. Grando R, Souza V H, Monteiro KM, Sousa IMO, Queiroz NCA, Carvalho JE, Spindola HM, Foglio MA. Comparison of Stantandardizes Dichloromethane viwth Aqueous Pterodon pubescens Benth. Fruit Extracts on Antinociceptive and AntiInflamatory Activity. J Braz Chem Soc [Internet]. 2017 [acesso em 12 de agosto de 2020]; 28(3):455-464. Disponível em: https://doi.org/10.21577/0103-5053.20160281

41. Dos Santos CBR, da Silva Ramos R, Ortiz BLS, da Silva GM, Giuliatti S, BalderasLopez JL, Navarrete A, Carvalho JCT. Oil from the fruits of Pterodon emarginatus Vog.: A traditional anti-inflammatory. Study combining in vivo and in silico. $\mathbf{J}$ Ethnopharmacol [Internet]. 2018 [acesso em 12 de agosto de 2020]; 222:107-120. Disponível em: https://doi.org/10.1016/j.jep.2018.04.041

42. Giorola, Leila Regina. Explorando a biodiversidade brasileira e os conhecimentos populares: desenvolvimento de formulação para uso tópico com extrato de Pterodon pubescens Benth [dissertação]. São Paulo: Instituto de Biologia da Universidade Estadual de Campinas; 2015. Disponível em: http://repositorio.unicamp.br/bitstream/REPO SIP/317699/1/Giarola_LeilaRegina_M.pdf 2013:272795. Disponível em:

43. Freitas PAB. Desenvolvimento de lipossomas com a superfície modificada por ácido hialurônico para aplicação tópica do extrato de Pterodon pubescens Benth [dissertação]. São Paulo: Faculdade de Engenharia Química da Universidade; 2019. Disponível em: http://repositorio.unicamp.br/bitstream/REPO SIP/335048/1/Freitas_PauloAdolfoBarboza_ M.pdf

44. Hansen D, Nakahata AM, Haraguchi M, Okamoto OK, Gonçalez E, Alonso A.14, 15epoxigeranilgeraniol e extratos isolados de Pterodon emarginatus vog. frutas: atividade antitumoral em células de glioblastoma. Journal of Applied Pharmaceutical Science [Internet]. 2012 [acesso em 12 de agosto de 2020]; 2(9):20-24. Disponível em: https://doi.org/10.7324/JAPS.2012.2904

45. Carvalho JCT. Fitoterápicos. Antiinflamatórios. Aspectos químicos, farmacológicos e aplicações terapêuticas. $1^{\mathrm{a}}$ ed., São Paulo: Tecmedd; 2004. p.384-400.

46. Menoita E, Santos V, Santos A, Gomes C. pH no controle do microambiente das feridas crónicas. Sinais Vitais [Internet]. 2011 [acesso em 12 de agosto de 2020]; 94:54-61. Disponível em: https://www.sinaisvitais.pt/index.php/revistasinais-vitais-publicacoes-78/revistas-19942014/24-revistas-2010-e-2012/513-revista-no94-janeiro-2011? showall=\&start=7

47. Hahn SW, Jung HS, Kim KS, Yun SH. Enhancing the transdermal penetration of nanoconstructis: could hyaluronic acid be the key. Nanomedicine [Internet]. 2014 [acesso em 12 de agosto de 2020]; 9(6):743-745. Disponível em: https://doi.org/10.2217/nnm.14.47

48. Vieira R, Severino P, Nalone LA, Souto SB, Silva AM, Lucarini M, Durazzo A, Santini A, Souto EB. Sucupira Oil-Loaded https://doi.org/10.31011/reaid-2021-v.95-n.36-art.1159 Rev Enferm Atual In Derme v. 95, n. 36, 2021 e-021153 
Nanostructured Lipid Carriers (NLC): Lipid Screening, Factorial Design, Release Profile, and Cytotoxicity. Molecules [Internet]. 2020 [acesso em 12 de agosto de 2020]; 25(3):685. Disponível em: https://doi.org/10.3390/molecules25030685

49. Nucci-Martins C, Martins DF, Nascimento LF, Venzke D, Oliveira AS, Frederico MJ, Silva FR, Brighente IM, Pizzolatti MG, Santos AR. Ameliorative potential of standardized fruit extract of Pterodon pubescens Benth on neuropathic pain in mice: Evidence for the mechanisms of action. $\mathbf{J}$ Ethnopharmacol [Internet]. 2015 [acesso em 12 de agosto de 2020]. Disponível em: https://doi.org/10.1016/j.jep.2015.09.005

50. Souza, VHS. Avaliação da toxicidade não clínica de extrato e vouacapanos obtidos dos frutos da espécie pterodon pubescens benth [tese]. São Paulo: Faculdade de Odontologia de Piracicaba da Universidade Estadual de Campinas; 2017. Disponível em: http://repositorio.unicamp.br/bitstream/REPO SIP/331400/1/Souza_VanessaHelenaDaSilva _D.pdf

\section{Autor Correspondente:}

Priscilla Roberta Silva Rocha

E-mail: priscillarocha@unb.br

Submissão: 2021-06-27

Aprovado: 2021-10-08 